\begin{tabular}{|c|l|}
\hline Title & Metal-induced gap states at well defined al kali-halide/metal interfaces. \\
\hline Author(s) & $\begin{array}{l}\text { Kiguchi, Manabu; A rita, Ry otaro; Y oshikawa, Genki; Tanida, Y oshiaki; Katay ama, Masao; Saiki, Koichiro; Koma, } \\
\text { A tsushi; A oki, Hideo }\end{array}$ \\
\hline Citation & $\begin{array}{l}\text { Physical Review Letters, 90(19), 196803 } \\
\text { https://doi.org/10.1103/PhysRevLet.90.196803 }\end{array}$ \\
\hline Issue Date & 2003-05-16 \\
\hline Doc URL & http://hdl.handle.net/2115/29774 \\
\hline Rights & Copyright $\odot$ 2003 A merican Physical Society \\
\hline Type & article \\
\hline File Information & PRL90-19.pdf \\
\hline
\end{tabular}

Instructions for use 


\title{
Metal-Induced Gap States at Well Defined Alkali-Halide/Metal Interfaces
}

\author{
Manabu Kiguchi, ${ }^{1}$ Ryotaro Arita, ${ }^{2}$ Genki Yoshikawa, ${ }^{3}$ Yoshiaki Tanida,${ }^{4}$ Masao Katayama, ${ }^{3}$ Koichiro Saiki, ${ }^{1,3}$ \\ Atsushi Koma, ${ }^{3}$ and Hideo Aoki ${ }^{2}$ \\ ${ }^{1}$ Department of Complexity Science and Engineering, Graduate School of Frontier Sciences, The University of Tokyo, \\ Hongo, Bunkyo-ku, Tokyo 113-0033, Japan \\ ${ }^{2}$ Department of Physics, Graduate School of Science, The University of Tokyo, Hongo, Bunkyo-ku, Tokyo 113-0033, Japan \\ ${ }^{3}$ Department of Chemistry, Graduate School of Science, The University of Tokyo, Hongo, Bunkyo-ku, Tokyo 113-0033, Japan \\ ${ }^{4}$ Fujitsu Laboratories Ltd., Atsugi, Kanagawa 243-0197, Japan
}

(Received 26 December 2002; published 15 May 2003)

\begin{abstract}
In order to search for states specific to insulator/metal interfaces, we have studied epitaxially grown interfaces with element-selective near edge x-ray absorption fine structure. An extra peak is observed below the bulk edge onset for $\mathrm{LiCl}$ films on $\mathrm{Cu}$ and $\mathrm{Ag}$ substrates. The nature of chemical bonds as probed by x-ray photoemission spectroscopy and Auger electron spectroscopy remains unchanged, so we regard this as evidence for metal-induced gap states (MIGS) formed by the proximity to a metal, rather than local bonds at the interface. The dependence on the film thickness shows that the MIGS are as thin as one monolayer. An ab initio electronic structure calculation supports the existence of the MIGS that are strongly localized at the interface.
\end{abstract}

DOI: 10.1103/PhysRevLett.90.196803

PACS numbers: 73.20.-r, 71.15.Mb, 73.40.Ns

Introduction. - While there is mounting interest in the nature of the "heterointerface" (solid-solid interfaces between very dissimilar materials), insulator/metal interfaces are especially intriguing, since they provide fascinating possibilities such as metal-insulator transition [1], band gap narrowing [2], and superconductivity [3], as well as technological possibilities such as catalysis, etc. Despite the interest, electronic structures characteristic of the insulator/metal interface have not been studied satisfactorily, for good reasons. First, well defined interfaces are hard to prepare due to the different nature of chemical bonds. Second, signals from the interface are obscured by significant contribution from the substrate in conventional experimental methods such as ultraviolet photoemission spectroscopy (UPS), inverse photoemission spectroscopy, or electron energy loss spectroscopy (EELS).

Recently, an interesting experimental result was reported for insulator/metal systems. Muller et al. studied $\{222\} \mathrm{MgO} / \mathrm{Cu}$ interfaces in fine particles (rather than in a film) with transmission EELS, and succeeded in observing metal-induced gap states (MIGS) for the first time [4]. The concept of MIGS was first introduced for semiconductor/metal junctions in discussing the Schottky barrier at the interface [5], and subsequently applied to insulator/metal interfaces [6]. MIGS are thought to accompany metal wave functions whose (exponential) tails penetrate into the insulating side of the interface. Schintke et al. studied $\mathrm{MgO} / \mathrm{Ag}(001)$ with scanning tunneling spectroscopy and found a state in the band gap [7]. However, the first point raised above remains: Since $\{222\} \mathrm{MgO}$ is a polar (hence presumably metallic) surface, whether the prepeak observed for $\{222\} \mathrm{MgO} / \mathrm{Cu}$ originates from the polar surface or from the MIGS has not been definite, although their study is pioneering. Further- more, $\{222\} \mathrm{MgO} / \mathrm{Cu}$ is not a well defined interface due to a large lattice mismatch. For $\mathrm{MgO} / \mathrm{Ag}(001)$, the strong hybridization between the $\mathrm{O} 2 p$ band and the $\mathrm{Ag} 5 s p$ band is expected to dominate the interface [8]. All these should obscure the identification of the MIGS in its proper sense, i.e., states formed solely by the proximity to a metal rather than by local bonds.

In order to clarify this, we propose here to prepare well defined interfaces by exploiting our studies, in which we have revealed that some alkali halides grow heteroepitaxially on metal substrates in a layer-by-layer fashion, so we end up with atomically well defined insulator/metal interfaces [9]. Being epitaxially grown, the number of atomic layers can also be controlled, which helps to probe the nature of the interface state. In a previous study we have examined the epitaxially grown $\mathrm{LiCl} / \mathrm{Cu}(001)$, but were unable to find an indication for the interface state [10], presumably because the methods employed (UPS and EELS) pick up signals from the substrate.

Here we adopt near edge $\mathrm{x}$-ray absorption fine structure (NEXAFS) to study electronic structures at the $\mathrm{LiCl} /$ metal interfaces, where the second point raised above comes in. Namely, we adopt $\mathrm{Cl}-K$ edge NEXAFS, which is based on $\mathrm{x}$-ray absorption by $\mathrm{Cl}$ atoms, and hence provides information on the $\mathrm{LiCl}$ film with negligible influences of the substrate. Furthermore, NEXAFS, with its high-energy photons, can probe very deep interfaces, so suited for obtaining the dependence of the interface states on the thickness of the insulating layer.

The results obtained here for $\mathrm{LiCl}$ thin films on $\mathrm{Cu}(001)$ and $\operatorname{Ag}(001)$ indeed exhibit clear evidence for the electronic states intrinsic to the insulator/metal interface which are as thin as one monolayer (ML) [1 ML = $2.6 \AA]$. We have then compared the experimental result with an $a b$ initio density functional calculation, which 
supports the existence of the MIGS that are strongly localized at the interface.

Experimental. - Epitaxial $\mathrm{LiCl}$ films were grown on metals at $300 \mathrm{~K}$ by a Knudsen cell. $\mathrm{Cl}-K$ edge NEXAFS was carried out at BL-11B in the Photon Factory in Institute of Materials Structure Science [11]. The Cl- $K$ NEXAFS provides information on the unoccupied $\mathrm{Cl}-p$ states[12].

Figure 1 shows the $\mathrm{Cl}-\mathrm{K}$ edge NEXAFS spectra for $\mathrm{LiCl} / \mathrm{Cu}(001)$ and $\mathrm{LiCl} / \mathrm{Ag}(001)$ taken at grazing $\mathrm{x}$-ray incidence $\left(15^{\circ}\right)$ for various thicknesses of the $\mathrm{LiCl}$ layer. All the spectra are normalized by their edge jumps. Two NEXAFS peaks are observed at $2827 \mathrm{eV}(p 1)$ and $2829 \mathrm{eV}$ $(p 2)$ for bulk LiCl. Now, a new finding here is that a pronounced prepeak ( $p 3)$ appears just below the bulk edge onset, which is clearly visible for thinner $\mathrm{LiCl}$ layers. Hereafter we will focus on the prepeak.

There are two points to note. First, the prepeak exists even for a $10 \mathrm{ML} \mathrm{LiCl}$ on $\mathrm{Cu}(001)$. Namely, although the peak may seem more prominent with decreasing the film thickness in Fig. 1, this is an artifact of normalizing the curve by the edge jump. As we shall elaborate in Fig. 1(c), the prepeak appears at $1 \mathrm{ML}$ and its intensity rapidly saturates. Second, the prepeak is observed for both $\mathrm{LiCl} / \mathrm{Cu}(001)$ and $\mathrm{LiCl} / \mathrm{Ag}(001)$. For $\mathrm{LiCl} / \mathrm{Cu}(001)$, $\mathrm{LiCl}$ grows with the [100] axis rotated by $45^{\circ}$ away from that of the $\mathrm{Cu}$ substrate, where both $\mathrm{Li}$ and $\mathrm{Cl}$ atoms sit on the fourfold hollow sites due to matched $\mathrm{Li}-\mathrm{Cl}$ and $\mathrm{Cu}-\mathrm{Cu}$ distances. On the other hand, $\mathrm{LiCl}$ on $\mathrm{Ag}(001)$ grows without an azimuthal rotation, for which a commensurate structure becomes impossible [9]. Despite the difference in the interface structure between $\mathrm{Cu}$ and $\mathrm{Ag}$, the prepeak is observed in both systems, which

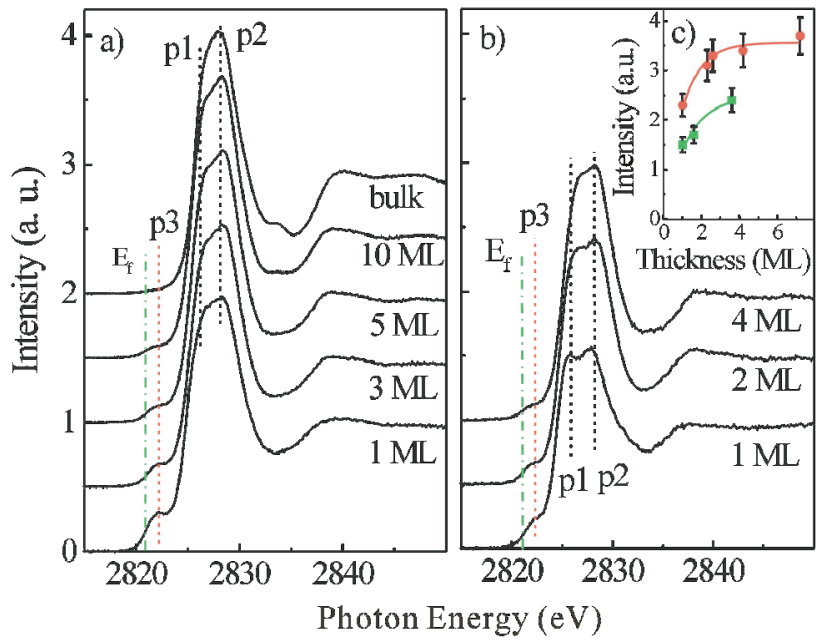

FIG. 1 (color). The Cl- $K$ edge NEXAFS spectra in $\mathrm{LiCl}$ films grown on (a) $\mathrm{Cu}(001)$ and (b) $\mathrm{Ag}(001) . E_{F}$ indicates the position of the Fermi level as determined from XPS. The inset (c) shows the intensity of the prepeak (not normalized by the edge jump unlike in the main panel) versus the film thickness, where the curves are the least-squares fit. precludes local structures at the interface as the origin of the prepeak.

We next confirm whether the nature of chemical bonds is modified around the interface by making use of $\mathrm{x}$-ray photoemission spectroscopy (XPS) and Auger electron spectroscopy (AES). For $\mathrm{LiCl} / \mathrm{Cu}(001)$, the $\mathrm{Cu} \mathrm{MMM}$ Auger peak does not shift from that of the clean $\mathrm{Cu}(001)$ within $0.1 \mathrm{eV}$, which implies that the interfacial $\mathrm{Cu}$ atoms remain almost neutral [13]. Figure 2 shows the $\mathrm{Cl} 1 s$ XPS spectra for $\mathrm{LiCl} / \mathrm{Cu}(001)$ for various thicknesses of $\mathrm{LiCl}$. For comparison, the spectrum for a bulk $\mathrm{LiCl}$ crystal and the peak position of a bulk $\mathrm{CuCl}$ crystal are also measured, which shows that the $\mathrm{Cl} 1 s$ binding energy in $\mathrm{LiCl}$ is larger by $2 \mathrm{eV}$ than that of $\mathrm{CuCl}$. If the $\mathrm{LiCl}$ film interacted with the $\mathrm{Cu}$ substrate to form new chemical bonds at the interface, a satellite peak at the lower binding energy side or the broadening of the peak would arise. However, both the binding energy and the width of the $\mathrm{Cl}$ $1 s$ peak do not change with the film thickness, nor does a satellite peak appear in $\mathrm{LiCl} / \mathrm{Cu}(001)$. These show that the chemical state of $\mathrm{Cl}$ in $\mathrm{LiCl} / \mathrm{Cu}(001)$ is virtually the same as that of bulk $\mathrm{LiCl}$ with no chemical bonds formed at the interface. Similar results are obtained from $\mathrm{Cl} 1 \mathrm{~s}$ XPS spectra for $\mathrm{LiCl} / \mathrm{Ag}(001)$.

Given the above NEXAFS, XPS, and AES results indicating that the prepeak does not originate from chemical bonds at the $\mathrm{LiCl} /$ metal interface, the gap states arise from the proximity to a metal, so we can regard them as MIGS. We can also note that the position of the Fermi

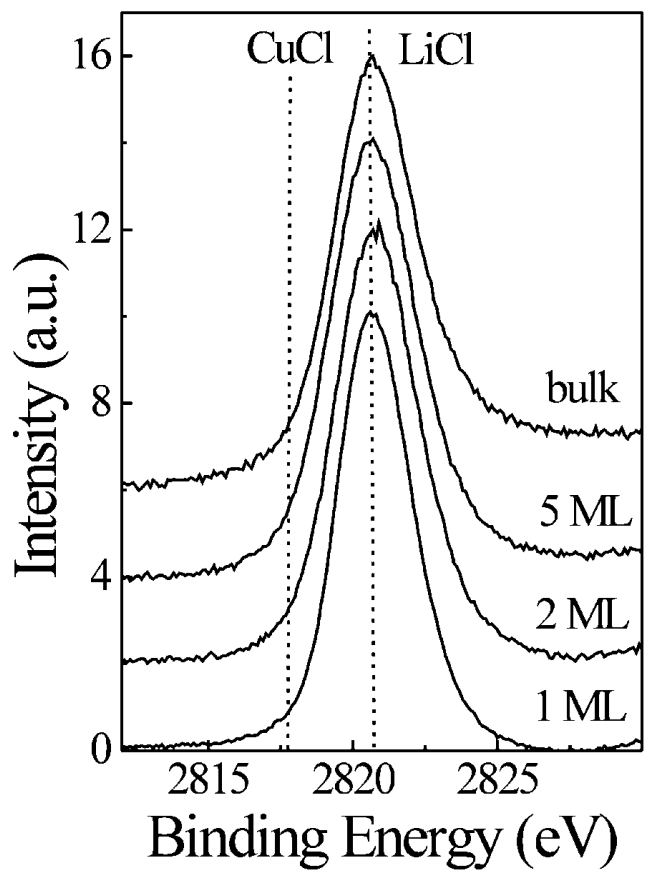

FIG. 2. The $\mathrm{Cl} 1 s$ XPS spectra in $\mathrm{LiCl} / \mathrm{Cu}(001)$ with a photon energy of $2900 \mathrm{eV}$. The binding energies are defined with respect to the Fermi level, where the binding energy of the bulk $\mathrm{CuCl}$ is also indicated. 
level, which can be estimated from the binding energy of $\mathrm{Cl} 1 s$ XPS spectra as indicated in Fig. 1, is seen to be located right in the prepeak. So a finite density of states at $E_{F}$ implies that $\mathrm{LiCl}$ is metallized at the interface.

Let us discuss the character of the MIGS in more detail from the NEXAFS data. First, we can exploit the epitaxygrown samples to estimate the decay length of the MIGS into the insulating side from the dependence of the intensity of the prepeak on the thickness of the insulating layer. Since the probing depth of NEXAFS is orders of magnitude greater (typically $\geq 10000 \AA$ ) than the atomic scale, NEXAFS intensity should directly reflect the decay of the MIGS into the insulator. If we assume an exponential decay to fit the experimental data, we end up with the decay length $2.6 \pm 0.3 \AA$ for $\mathrm{LiCl} / \mathrm{Cu}(001)$ and $2.9 \pm 0.7 \AA$ for $\mathrm{LiCl} / \mathrm{Ag}(001)$ [Fig. 1(c)], so the MIGS are indeed localized within a few $\AA$ (approximately as small as one monolayer) of the interface.

Second, we have probed the geometry of the MIGS wave function from the polarization dependence of NEXAFS. The prepeak has turned out to be greater for grazing X-ray incidence (Fig. 3), which clearly shows that the MIGS are $p_{z}(z \perp$ surface)-like[14].

Ab-initio calculation. - Formation of MIGS with a thickness $\sim 1 \mathrm{ML}$ is theoretically intriguing, since a simple model for the interface (e.g., tight-binding insulator/jellium) would predict a negligible ( $\ll 1 \mathrm{ML})$ penetration (for the gap of the insulator $\sim$ several $\mathrm{eV}$ )

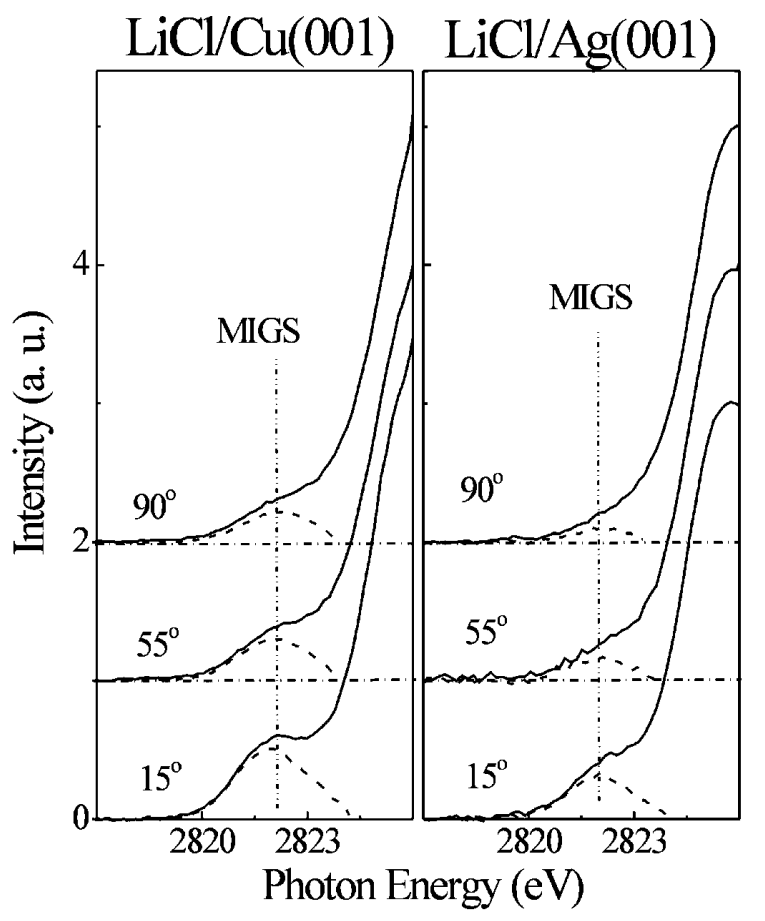

FIG. 3. Polarization dependence of the $\mathrm{Cl}-K$ edge NEXAFS spectra for $1 \mathrm{ML} \mathrm{LiCl} / \mathrm{Cu}(001)$ and $1 \mathrm{ML} \mathrm{LiCl} / \mathrm{Ag}(001)$. The MIGS-derived peaks (difference between the spectra for bulk $\mathrm{LiCl}$ and $\mathrm{LiCl} / \mathrm{Cu}$ ) are indicated by dotted lines. and would completely fail. So we have performed a first-principles calculation based on the local density functional theory (LDF) with pseudopotentials and plane-wave basis. We have adopted two models, one for $\mathrm{LiCl} / \mathrm{Cu}(001)$ and the other for $\mathrm{LiCl} /$ jellium, to examine whether the MIGS come from the specific metallic substrate (copper here) or a general property of the proximity to an electron gas. The calculation for $\mathrm{LiCl} / \mathrm{Cu}$ is performed on periodically repeated slabs with five $\mathrm{Cu}$ layers sandwiched from top and bottom by one $\mathrm{LiCl}$ layer each with the slabs separated by a vacuum of height $50 \AA$. The in-plane unit cell contains two $\mathrm{Cu}$ atoms and one $\mathrm{LiCl}$, which is a virtue of the commensurate structure.

Figure 4(a) shows the band structure of $1 \mathrm{ML}$ $\mathrm{LiCl} / \mathrm{Cu}(001)$. For comparison we have also obtained the band structure of an isolated $1 \mathrm{ML} \mathrm{LiCl} \mathrm{film} \mathrm{without}$ the metallic substrate. The isolated $\mathrm{LiCl}$ has a large band gap, although the size of the gap is, as usually the case with LDF, underestimated by $2-3 \mathrm{eV}$. When the $1 \mathrm{ML} \mathrm{LiCl}$ is put on $\mathrm{Cu}(001)$, new bands appear in the gap. We have characterized their nature by looking at the amplitude of the LDF wave functions.

If we look at the three states just above $E_{F}$ at $\Gamma$ point, the two in-gap bands closest to $E_{F}$ have indeed amplitudes that are localized at the interfacial $\mathrm{Cl}$ and $\mathrm{Cu}$ atoms [Fig. 4(b)]. Existence of such states is, theoretically, remarkable given the fact that $\mathrm{LiCl}$ is a very good insulator. They are $p_{z}$-like, in agreement with the polarization dependence seen in NEXAFS. Thus we identify that the MIGS observed by NEXAFS should correspond to these states.
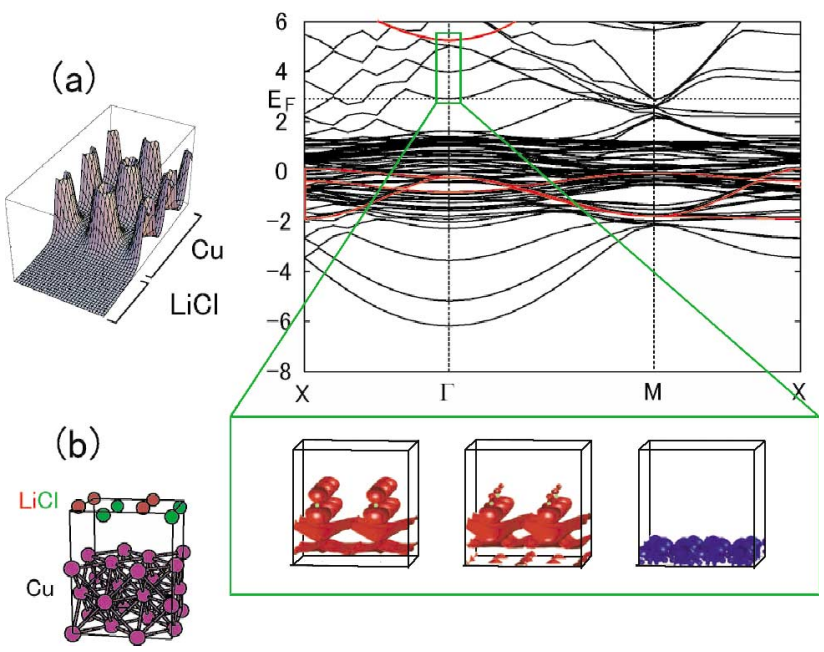

FIG. 4 (color). (a) The band structure of $1 \mathrm{ML} \mathrm{LiCl} / \mathrm{Cu}(001)$ (black) as compared with that for an isolated $1 \mathrm{ML} \mathrm{LiCl}$ (red). (b) Contours of the absolute value of the LDF wave functions for the in-gap states (in the ascending order of energy, enclosed in green), along with the atomic configuration. The wave functions having amplitudes on both $\mathrm{Cl}$ and $\mathrm{Cu}$ atoms are shown in red. The top-left inset shows the charge distribution difference between $\mathrm{LiCl} / \mathrm{Cu}(001)$ and an isolated $\mathrm{LiCl}$. 
(a)

(b)
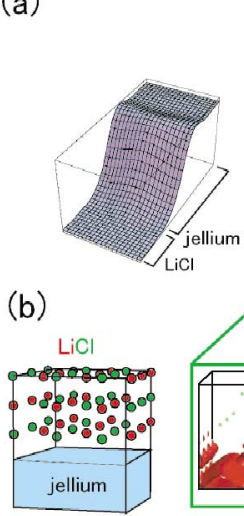

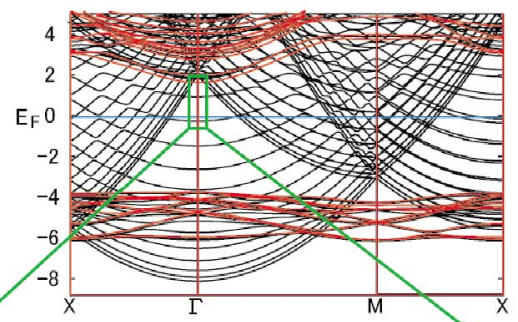

$x$

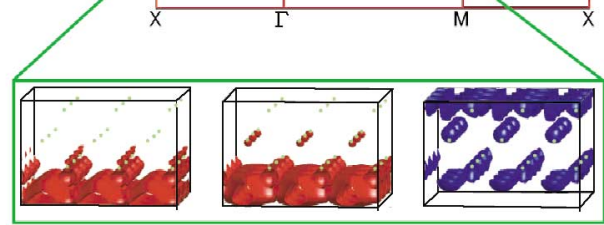

FIG. 5 (color). (a) The band structure of $3 \mathrm{ML} \mathrm{LiCl} /$ jellium with $r_{s}=2.5$ (black) as compared with that for an isolated $3 \mathrm{ML} \mathrm{LiCl}$ (red). (b) Contours of the absolute value of the LDF wave functions, along with the atomic configuration. The wave functions having amplitudes on both $\mathrm{Cl}$ and jellium are shown in red. The top-left inset shows the charge distribution difference between $\mathrm{LiCl} /$ jellium and an isolated $\mathrm{LiCl}$.

Another theoretical observation is that the new state is by no means a result of a charge transfer across $\mathrm{Cu}$ and $\mathrm{LiCl}$. This can be seen in the inset of Fig. 4, which displays the difference in the charge density between $\mathrm{LiCl} / \mathrm{Cu}(001)$ and the isolated $\mathrm{LiCl}$ film. If there were a charge transfer, the charge difference would have an amplitude on the $\mathrm{LiCl}$ side as well, while the result shows that it is entirely confined to the $\mathrm{Cu}$ side. The absence of charge transfer can explain the absence of core level shifts at the interface in $\mathrm{Cl} 1 s$ XPS result (Fig. 2).

In order to theoretically estimate the decay length of the MIGS into the insulator, we have then performed an LDF calculation for $3 \mathrm{ML} \mathrm{LiCl}$ attached to a jellium of thickness $80 \AA$ (from top and bottom). The replacement of the $\mathrm{Cu}$ substrate with a jellium also enables us to examine whether a simplest possible model for the electron gas would already exhibit MIGS. Figure 5(a) shows the band structure of $3 \mathrm{ML} \mathrm{LiCl} /$ jellium, where the jellium has $r_{s}=2.5$. We have again new in-gap states whose wave functions [Fig. 5(b)] just extend to the interfacial (i.e., the first layer from the jellium) $\mathrm{Cl}$ atoms. This result for $\mathrm{LiCl} /$ jellium endorses that (i) MIGS are characteristic of insulator/metal interfaces rather than of local chemical bonds, and (ii) $\sim 1$ ML penetration depth of the MIGS is rather general.

To systematically confirm how the MIGS appear, we have then varied the density of electrons in the metallic side by varying $r_{s}$, which is the sole parameter characterizing the jellium. The LDF result shows that MIGS appear with its penetration depth only weakly dependent on $r_{s}$. This accounts for the experimental result that MIGS are observed for both $\mathrm{Cu}$ (with $\left.r_{s}=2.7\right)$ and $\mathrm{Ag}\left(r_{s}=3.0\right.$ ) substrates.

Finally, let us point out that the electronic structure specific to insulator/metal interfaces can possibly have interesting implications for superconductivity. Discussions on superconductivity at interfaces have a long history. A well known proposal is by Ginzburg et al. [3] for the possibility of superconductivity by exciton mechanism at metal/semiconductor interfaces. The presence of excitons requires a wide-gap insulator, which will, however, prevent the metallic carriers to penetrate into the insulator, so that the coexistence (in real space) of excitons and metallic carriers is difficult. To circumvent this they [3] had to consider a hypothetical density of states that has two closely separated peaks in a wide band gap, where the former are required to enable the carriers in the metallic side to tunnel into the semiconductor side.

By contrast, in the present MIGS in insulator/metal interfaces we automatically have a coexistence of excitations [associated with the wide band gap $(\simeq 9 \mathrm{eV}$ for $\mathrm{LiCl})$ of the insulator] and carriers. To realize a high $T_{C}$ we have further to realize strong interaction between the carriers and excitons, which should also be fulfilled due to the penetration of MIGS into the insulating layer. So we may envisage that the insulator/metal interface as discussed here may provide a possible ground for superconductivity.

This work was supported by a Grant-in-Aid for Creative Scientific Research, Grant No. 14GS0207, MEXT, and PF-PAC (Grant No. 2001G336). The numerical calculation was performed with SR8000 in ISSP, University of Tokyo.

[1] For a review, see P.W. Anderson, in Elementary Excitation in Solids, Molecules and Atoms, Part A, edited by J. A. Devreese et al. (Plenum Press, New York, 1974), p. 1.

[2] R. Arita et al., Phys. Rev. B 64, 245112 (2001).

[3] High-Temperature Superconductivity, edited by V.L. Ginzburg and D. A. Kirzhnits (Consultants Bureau, New York, 1982).

[4] D. A. Muller et al., Phys. Rev. Lett. 80, 4741 (1998).

[5] J. Tersoff, Phys. Rev. Lett. 56, 2755 (1986).

[6] M.W. Finnis, J. Phys. Condens. Matter 8, 5811 (1996).

[7] S. Schintke et al., Phys. Rev. Lett. 87, 276801 (2001).

[8] S. Altieri et al., Phys. Rev. B 59, R2517 (1999).

[9] M. Kiguchi et al., Phys. Rev. B 66, 155424 (2002).

[10] M. Kiguchi et al., Surf. Sci. 522, 84 (2002).

[11] T. Ohta et al., Nucl. Instrum. Methods Phys. Res., Sect. A 246, 373 (1986).

[12] Although $1 \mathrm{~s}$ core holes might distort the position and intensity of near edge features, they are assumed to introduce no new features within the present energy resolution $(1.5 \mathrm{eV})$. This assumption has been verified for graphite (C- $K$ edge) and even for localized electrons in $\mathrm{MgO}$ (O-K edge) [4]; X. Weng et al., Phys. Rev. B 40, 4175 (1989). This allows a single-particle interpretation of the NEXAFS spectra.

[13] M. P. Hooker et al., J. Vac. Sci. Technol. 12, 325 (1975).

[14] NEXAFS Spectroscopy, edited by J. Stöhr (SpringerVerlag, Berlin, 1992). 